Bangladesh J. Plant Taxon. 13(1): 63-68, 2006 (June)

\title{
ETHNOBOTANICAL SURVEY OF MEDICINAL PLANTS IN PHULBARI UPAZILA OF DINAJPUR DISTRICT, BANGLADESH
}

\author{
Mohammad Zashim Uddin, Md. Abul Hassan and Mahmuda Sultana \\ Department of Botany, University of Dhaka, Dhaka-1000. Bangladesh
}

Key words: Ethnobotanical survey, threats and conservation, Bangladesh

\begin{abstract}
Ethnobotanical survey in Phulbari Upazila of Dinajpur district has revealed a total of 86 species used as medicinal plants by the Santal community. Santal names, part/s used as medicine and diseases to be treated with each plant have been presented. A number of threats to medicinal plants and their habitats have been identified and some measures have also been recommended for the conservation of medicinal plants and their habitats in the area.
\end{abstract}

\section{Introduction}

Phulbari Upazila belongs to Dinajpur district. It lies between $250^{\circ} 23^{\prime}$ and $25^{\circ} 34^{\prime} \mathrm{N}$ latitude and 88 $88^{\prime}$ and $88^{\circ} 59^{\prime}$ E longitude. The Upazila is bounded by Parbotipur and Shiribandar to the north, by Nawabganj to the east, by Birampur to the south and east and by India to the west. Total area of the Upazila is about 299.55 sq. km. The general topography of the Upazila may be described as flat, gently sloping southward and slightly elevated alluvial terrace known as Barind. Elevation ranges from 25 to 35 meters above mean sea level (Siddiqi 1972).

Once maximum area of the Upazila was occupied by an extensive Sal (Shorea robusta Gaertn.) forest interspersed with cultivated rice fields. Due to human settlement, agricultural encroachment and mining activities, the Sal forest of the area has been drastically reduced to small patches. In the small patches of the forest, $S$. robusta is the dominant species. Some other species associated with the Sal are Careya arborea (Kumbhi), Anacardium occidentale (Bela), Cassia fistula (Sonalu), Albizia procera (Koroi). Syzygium fruticosum (Butijam), Syzygium operculatum (Panijam), Syzygium cumini (Kalojam), Flacourtia indica (Paniala), Randia dumetorum (Monkanta), and Litsae glutinosa (Menda). Forest floor has been covered with seasonal vegetation including grasses, sedges, aroids, zingers, climbers, herbs etc.

Phulbaria Upazila is the abode for 1.3 million human population (Asiatic Society of Bangladesh 2003). Among this, 3.11\% population belongs to Santal community They are living in the forest sites far from the Upazila headquarters. A major share of their food, medicine, house buildings materials and firewood come from the natural forest. These people have their own language and cultural tradition. They always like to keep away from the hub of modern civilization. Currently, their cultural tradition is threatened by 
modern cultures all around them. They already started to convert to Christianity from Hinduism. They are losing their traditional knowledge day by day. Apart from this, mining activities and forest clearance around their home sites are other major threats to their traditional culture. Considering all these factors ethnobotanical survey of medicinal plants in Phulbari Upazila will require much time to complete. Otherwise we may lose important traditional Santal knowledge about plants before documentation.

Ethnobotanical work here in Bangladesh is in its initial stage. Some work, e.g. Hassan and Khan (1986), Mia and Huq (1988), Hassan and Khan (1996), Chowdhury et al. (1996), Alam et al. (1996), Uddin et al. (2001). Khan et al. (2002) and Uddin et al. (2004) are only a few to mention. The work on the ethnobotany of Santal community is lacking. That is why in the present survey an attempt has been made with the following objectives:

1) To identify the medicinal plants, their Santal names, parts used and diseases to be treated

2) To identify the threats to medicinal plants and their habitats

3) To make recommendation for conservation measures.

\section{Materials and Methods}

Phulbari Upazila of Dinajpur District was selected for the study and Santal community was considered as target community. All Santal villages in the Upazila were visited during the year of 2004 and 2005. Data of medicinal use of plants were collected through interview with local herbal practitioners (Kabiraj/ Boidya), headmen and elderly persons in the community using semi-structured questionnaire at different locations. Data collected from one person were verified with others by asking the same questions. Most of the medicinal plants were identified in the field and in case of unknown, plant specimens were collected. These specimens were brought to Dhaka University Herbarium and processed by traditional herbarium techniques. These were examined and identified by comparing herbarium specimens and also consulting literature. Threats to medicinal plants and their habitats were also noted from the field observations.

\section{Results and discussion}

A total of 86 medicinal plant species were recorded from the present survey work in Phulbari Upazila. These species are used by Santal community in different ailments. Botanical names, Santal names, parts used and diseases to be treated are presented in the Table 1.

Currently, coal mining, stone lifting and related developmental activities in Phulbari Upazila are great threats to medicinal plants and their habitats. Moreover, Santal

community already started to convert themselves to Christianity. Missionary activities 
gave them opportunity to go for modern medicine. It was found that many medicine men are reluctant to go back to Santal community and their traditional health care system. Forest clearance for exotic monoculture plantations in Phulbari Upazila is other threat to indigenous medicinal plants. Sal forest with associated species were replaced by Acacia spp. and Eucalyptus spp. plantations in different natural forest patches of the Upazila. Remaining Sal patches are in great risk because of fragmentation, edge effect, agricultural encroachment and developmental activities.

From the present observation in the Phulbari Upazila, we have come up with some recommendation measures for the conservation of medicinal plants and their habitat. Traditional Santal knowledge about the usage of medicinal plants should be properly recorded and documented. Apart from several threats some Sal patches of the Upazila still merit for in situ conservation. Otherwise ex-situ conservation sites including medicinal plant garden, protected area and eco-park should be established. Awareness about the importance of medicinal plants should be created among the local people, developers, energy companies and policy makers. Environmental impact assessment should be done before going to undertake any mining and developmental projects. Compensation measures should be ensured from companies for damaging the medicinal plants and their habitats.

Table 1. List of medicinal plants used by Santal community of Phulbari Upazila under Dinajpur district.

\begin{tabular}{|c|c|c|c|}
\hline Scientific name & Santal name & Parts used & Diseases to be treated \\
\hline Achyranthes aspera L. & Kakra lata & Root & Jaundice \\
\hline Aegle marmelose Corr. & Singadare & Fruits & Laxative urinary diseases \\
\hline Agave Americana L. & Kongak & Leaves & Ear lesion \\
\hline Albizia procera Benth. & Koroi & Leaves & Allergy \\
\hline Alstonia scholaris L. & Chatinidare & Bark & Aphrodisiac, Impotence \\
\hline Amaranthus spinosus L. & Jenumara & Whole plant & Chest pain \\
\hline Amaranthus virdis L. & Gandareshak & Whole plant & Vegetable \\
\hline Anacardium occidentale L. & Shasho & Fruits & Mump, antiseptic \\
\hline $\begin{array}{l}\text { Andrographis paniculata (Burm.f.) } \\
\text { Wall. }\end{array}$ & Chirata & Whole plant & Malarial fever \\
\hline Anisomeles indica (L.)O. Kuntz & Kukurmuta & Fruits & Impotence \\
\hline Antidesma ghaesembila Gaertn. & Chudumathasune & Leaves & Fever \\
\hline Azedirachta indica A. Juss. & Neemdare & Leaves & $\begin{array}{l}\text { Fever, malaria, lesion, } \\
\text { abscess }\end{array}$ \\
\hline Biscofia javanica $\mathrm{Bl}$. & Mathasure & Leaves & Kidney diseases \\
\hline Bombax ceiba L. & Edaldare & Root & Impotance \\
\hline Borreria articularis (L.f.) Williams. & Mudmala & Leaves & Eye pain \\
\hline Caesalpinia crista L. & Baghinjanum & Fruit,seed & $\begin{array}{l}\text { Headache, color for } \\
\text { fishing net }\end{array}$ \\
\hline Cardiospermum helicacavum L. & Chatolature & Stem & Heart pain \\
\hline
\end{tabular}


Table 1. (Contd.)

\begin{tabular}{|c|c|c|c|}
\hline Caryea arborea Roxb. & Kumbidare & Bark & Weakness \\
\hline Cassia fistula $\mathrm{L}$. & Neduic & Leaves, fruits & Ring worm, laxative \\
\hline Centella asiatica Urban. & Dolbamon & Whole plant & Gastric \\
\hline Cissus adnata Roxb. & Bodlar & Stem & Paralysis \\
\hline Clerodendrum viscosum Vent. & Banni & Roots,leaves & Healing cut injury, fever \\
\hline Commelina bengalensis L. & Jeotin & Root & Menstrual disorder \\
\hline Crinum asiaticum L. & Birpiaj & Root & Ringworm \\
\hline Curculigo orchioides Gaertn. & Birparo & Root & Healing, cut injury \\
\hline Curcuma longa L. & Shasang & Rhizome & Blood purifier \\
\hline Curcuma zedoaria (Christm) Rosc. & Pado & Rhizom & Diarrhoea \\
\hline Cuscuta reflexa Roxb. & Alakgudi & Wholeplant & $\begin{array}{l}\text { Rheumatic fever, } \\
\text { Lesion, Jaundice }\end{array}$ \\
\hline Cynodon dactylon L. & Dubigass & Wholeplants & Healing cut injury \\
\hline Cyperus rotundus Vahl. & Takudare & Root & Paralysis \\
\hline Dioscorea bulbifera L. & Damru & Root & Fever, Krimi, vegetable \\
\hline Elephantopus escaber L. & Ranurang & Roots & Abscess \\
\hline Erythrina veriegata $\mathrm{L}$. & Mararbaha & Flower & Waist Pain \\
\hline Eupatorium odoratum L. & Randai & Leaves & Healing cut injury \\
\hline Euphorbia hirta L. & Kushitoa & Whole plant & Head injury \\
\hline Euphorbia thymifolia Burm. f. & Gutedare & Leaves & Waist pain \\
\hline Ficus racemosa L. & Loa & Fruits & Krimi, Blood purifier \\
\hline $\begin{array}{l}\text { Glochidion multiloculare (Roxb. } \\
\text { ex.Willd.) Muell.-Arg. }\end{array}$ & Kudurpala & Leaves,root & Diarrhea of cow \\
\hline Glycosmis pentaphylla (Retz.) A. DC. & Atishadha & Stem & Jaundices, Tooth brush \\
\hline $\begin{array}{l}\text { Holarrhena pubescens (Buch.-Ham) } \\
\text { Wall. ex. G. Don. }\end{array}$ & Hartdare & Bark & Diarrhoea, dysentery \\
\hline Hyptis sauveolens(L.)Poit. & $\begin{array}{l}\text { Kukurmuta } \\
\text { (Sada) }\end{array}$ & Fruits & Impotence \\
\hline Indigofera tinctoria L. & Nildare & Root & Ulcer \\
\hline Jatropha curcas L. & Kuruzdare & Fruits & Lesion, ring worm \\
\hline Jatropha gossipyfolia L. & Beddha & Leaves & Dysentery \\
\hline Lannea coromandelica (Houtt.) Merr. & Dokadare & Bark & Diarrhea \\
\hline Leea macrophylla Roxb. & Harmadare & Root & Healing cut injury \\
\hline Leportia crenulata Gaud. & Sengelsingh & Root & Head ache \\
\hline Litsea glutinosa (Lour.) C.B. Robinson & Maliata & Leaves,bark & $\begin{array}{l}\text { Diarrhoea, dysentery, } \\
\text { aphrodisiac }\end{array}$ \\
\hline $\begin{array}{l}\text { Mallotus philippensis ( Lamk.) Muell.- } \\
\text { Arg. }\end{array}$ & Ruda & Barks & Piles \\
\hline Mangifera indica L. & Uldare & Bark,leaves & Diarrhoea \\
\hline Merrimia umbellata (L.) Hallier.f. & Haruamar & Stem & Indigestion \\
\hline Mimosa pudica L. & Japhi & Root & Impotence, aphrodisiac \\
\hline Mimosa rubricaulis Lamk. & Kondrajenure & Root & $\begin{array}{l}\text { Impotence, Menstrual } \\
\text { disorder }\end{array}$ \\
\hline Moringa olifera Lamk. & Munga & Bark & To refrain from snake \\
\hline
\end{tabular}


Table 1 (Contd.)

\begin{tabular}{|c|c|c|c|}
\hline Mucuna prurins (L.) DC. & Bandoneri & Stem & Waist pain \\
\hline Murraya koenigii Spreng & Jimtidare & Leaves & Menstrual disorder \\
\hline Ocimum sanctum L. & Torshi & Leaves & Fever, bronchitis \\
\hline Oroxylum indicum (L.) Kurz. & Banahata & Bark,fruit & Jaundice, cow diseases \\
\hline Persicaria hyropiper (L.) Spach. & Jeoti & Root & Impotence \\
\hline Phyllanthus emblica L. & Lodam & Fruits & Jaundice, diarrhoea \\
\hline Phyllanthus reticulatus Poir & Simikdare & Stem & Tooth brush \\
\hline Pterospermum acerifolium Willd. & Moskanda & Flower & Brain treatment \\
\hline Ricinus communis L. & Araddom & Bark,fruit & Eye treatment \\
\hline Scoparia dulcis L. & Sinipata & Leaves & Diarrhoea \\
\hline Senna accidentalis (L.) Link. & Junjunea & Leaves & Diabetes \\
\hline Senna sophera (L.) Link. & Bedatheri & Root & Lesion \\
\hline Senna tora (L.) Roxb. & Sakamenda & Root & Indigestion \\
\hline Shorea robusta Gaertn. & Sajamdare & Bark,root & Menstrual disorder \\
\hline Sida acuta Burm. f. & Sipsedip & Leaf & Head ache \\
\hline Sida cordata (Burm.f) Borss. & Japkhasakam & Leaf & Abscess \\
\hline Smilax zeylanica L. & Katrupala & Root & Menstrual disorder \\
\hline Solanum nigram L. & Hedikudi & Leaves & Eye disease \\
\hline Solanum torvum S.W. & Bengar & Fruits & Hopping Cough, ear rotten \\
\hline Stephania japonica (Thunb.) Miers. & Tezomala & Stem & Jaundice, foot rot of cow \\
\hline Sterculia foetida L. & Sekra & Bark, pellicles & Impotence, weakness, tonic \\
\hline Streblus asper L. & Sharha & Bark & Pain, Diarrhoea \\
\hline Suregada multiflora (A. Juss.) Baill. & Charchu & Fruit & Fish kill \\
\hline $\begin{array}{l}\text { Terminalia arjuna (Roxb. ex. DC.) Wt. } \\
\text { and Arn. }\end{array}$ & Arjun & Barks & Heart diseases \\
\hline Terminalia belerica Roxb. & Lopung & Fruits & Menstrual disorder \\
\hline Terminalia chebula Retz. & Rol & Fruits & Dysentery \\
\hline $\begin{array}{l}\text { Trichosanthes bracteata (Lamk.) } \\
\text { Voigt. }\end{array}$ & Kahubutki & Root & Gastric paid \\
\hline Typhonium trilobatum Schott. & Nirbish & Leaves & Constipation \\
\hline Urena lobata L. & Bedijone & Root & Lesion \\
\hline Vernonia patula Merrill. & Shandani & Root & Menstrual, disorder \\
\hline Zizyphus mauritiana Lamk. & Jenumdare & Leaves & Headache \\
\hline Zizyphus xylopyrus (Retz.)Willd. & Sekera & Bark & Constipation \\
\hline
\end{tabular}

\section{Ackonwledgement}

The authors are highly grateful to SMEC (Snow Mountain Environmental Corporation, Australia) for financial support for the field work.

\section{References}

Alam, M.K. 1992. Medical ethnobotany of Marma tribe of Bangladesh. Economic Botany 46 (3): 330-335.

Alam M.K., Chowdhury, J. and Hassan, M.A. 1996. Some folk formularies from Bangladesh J. Life Sci. 8(1): 49-63 
Asiatic Society of Bangladesh 2003. Banglapedia (Ed. Sirajul Ialam and Sajahan Mia), 8: 73-74.

Chowdhury, J., Alam, M.K. and Hassan, M.A. 1996. Some folk formularies against dysentery and diarrhoea in Bangladesh. J. Econ. Taxon. Bot. Additional series 12, Scientific Publishers Jodhpur (India), pp. 20-23.

Hassan, M.A. and Khan, M.S. 1986. Ethnobotanical record in Bangladesh-1. Plant used for healing fractured bones. J. Asiatic Society, Bangladesh (Sci.) 12(IA2): 33-39.

Hassan, M.A. and Khan, M.A.1996. Ethno botanical record in Bangladesh-2 . Plants used for healing cut's and wounds. Bangladesh J. Plant Taxon. 3(2): 49-52

Khan, M.S, Hassan, M.A and Uddin, M.Z. 2002. Ethnobotanical survey in Rema Kalenga Wildlife Sanctuary (Habiganj) in Bangladesh. Bangladesh J. Plant Taxon. 9(1): 51-60.

Mia, M.M.K. and Huq. A.M, 1988. A preliminary ethnobotanical survey in the Jointiapure, Tamabil and Jafflong area, Sylhet, Bangladesh National Habarium Bull. 3, pp.1-10.

Siddiqi, A. 1972. Bangladesh District Gazetteers for Dinajpur, Bangladesh Government Press, Dhaka, pp. 4-3.

Uddin, M.Z., Khan, M.S. and Hassan, M.A. 2001. Ethnobotanical plant records of Kalanga forest range (Habiganj), Bangladesh for malaria, jaundice, diarrhoea and dysentery. Bangladesh. J. Plant Taxon. 8(1): 101-104

Uddin, S.N, Uddin, M.Z, Hassan, M.A and Rahman, M.M.2004. Preliminar ethnomedical plant survey in Khagrachari district, Bangladesh. Bangladesh J. Plant Taxon. 11(2): 39-48. 